\title{
Estudio del grado de satisfacción en pacientes edéntulos totales tratados con implantes.
}

\author{
Regina Mestre Aspa * \\ Ma Àngels Sánchez Garcés * \\ Leonardo Berini Aytés * \\ Cosme Gay Escoda *
}

\begin{abstract}
Regina Mestre Aspa, $\mathrm{M}^{\mathrm{a}}$ Àngels Sánchez Garcés, Leonardo Berini Aytés, Cosme Gay Escoda. Estudio del grado de satisfacción en pacientes edéntulos totales tratados con implantes. Av Periodon Implantol. 2001; 13, 2: 93-99.
\end{abstract}

\begin{abstract}
RESUMEN
El objetivo de este estudio fue evaluar el grado de satisfacción de los pacientes edéntulos totales tratados en nuestro servicio mediante implantes dentales y la colocación posterior de una prótesis implantosoportada (sobredentadura o prótesis fija). Para evaluar el grado de satisfacción se realizó una encuesta telefónica con seis preguntas: 1) ¿Siente algún tipo de dolor en los implantes? 2) ¿Tiene algún problema cuando habla? 3) ¿Se atreve a reír con esta prótesis? 4) ¿Puede comer cómodamente? 5) ¿Está satisfecho en general con el tratamiento recibido y el resultado de éste? 6) ¿Repetiría el tratamiento?. Para cuantificar la satisfacción de los pacientes se valoraron de forma individual y de forma conjunta las respuestas a estas preguntas.
\end{abstract}

De los datos obtenidos destacaremos que el $65 \%$ de los pacientes encuestados estaban satisfechos con el tratamiento recibido y con el resultado de éste, independientemente de si estos eran portadores de una prótesis fija o de una sobredentadura. También el $65 \%$ de los pacientes repetirían el tratamiento. Mediante el test de c2 (grado de significación p $<0.05$ ) se relacionaron las diferentes variables (tipo de prótesis implantosoportada y tipo de dentición presente en la arcada antagonista) con el grado de satisfacción de los pacientes.

\section{PALABRAS CLAVE}

Satisfacción, implantes dentales, sobredentadura, prótesis fija.

\section{INTRODUCCIÓN}

La reabsorción de la cresta alveolar que sigue a la pérdida de dientes comporta una falta de estabilidad y de retención de las prótesis completas convencionales, reduciendo a su vez el confort de los pacientes, su habilidad masticatoria y para hablar, y su estética. Todos estos problemas acaban provocando una baja autoestima en la mayoría de los pacientes (1-3).
Casi todos los trabajos y estudios implantológicos hacen referencia a su eficacia, su biocompatibilidad, su diseño, su osteointegración, y sus resultados, pero pocos nos informan sobre el grado de satisfacción de los pacientes en relación con los tratamientos implantológico y prostodóncico recibidos.

Para muchos pacientes el tratamiento con implantes osteointegrados ofrece una importante mejora en la

\footnotetext{
* Odontóloga. Alumna del Máster de Cirugía e Implantología Bucal. Facultad de Odontología de la Universidad de Barcelona.

** Médica Estomatóloga. Profesora asociada de Cirugía Bucal. Profesora del Máster de Cirugía e Implantología Bucal. Facultad de Odontología de la Universidad de Barcelona.

*** Profesor titular de Patología Quirúrgica Bucal y Maxilofacial. Profesor de Máster de Cirugía e Implantología. Facultad de Odontología de la Universidad de Barcelona.

**** Catedrático de Patología Quirúrgica Bucal y Maxilofacial. Director del Máster de Cirugía e Implantología. Facultad de Odontología de la Universidad de Barcelona. Cirujano maxilofacial del Centro Médico Teknon. Barcelona.
} 
rehabilitación prostodóncica y en la estabilidad y retención de las prótesis. En pacientes portadores de prótesis completas convencionales que, posteriormente, han sido rehabilitados con una prótesis, tipo sobredentadura, implantosoportada, incluso se ha podido ver un aumento significativo en los niveles de fuerza oclusal y unos cambios funcionales satisfactorios (4). Además de sobredentaduras también podemos ofrecer a nuestros pacientes la posibilidad de colocar una prótesis fija sobre implantes; sin embargo, existen estudios en los que se ha comprobado que no existen diferencias significativas en la satisfacción de los pacientes edéntulos totales portadores de sobredentaduras y los portadores de prótesis fijas, ambas implantosoportadas $(5,6)$.

El objetivo de este trabajo fue evaluar el grado de satisfacción de este tipo de pacientes edéntulos tratados en nuestro servicio mediante implantes dentales y a los que posteriormente se realizó una prótesis implantosoportada (fija o sobredentadura).

\section{PACIENTES Y MÉTODO}

Fueron incluidos en el estudio los pacientes edéntulos totales intervenidos entre los años 1991 y 1998 en la Unidad de Implantología Bucofacial del Máster de Cirugía e Implantología Bucal de la Universidad de Barcelona.

Se registró en cada paciente el tipo de edentulismo total (uni o bimaxilar), la edad, el sexo, el tipo de prótesis implantosoportada colocada y el tipo de dentición de la arcada antagonista.

El método de evaluación del grado de satisfacción de los pacientes consistió en una encuesta telefónica personal con seis preguntas rápidas y de fácil respuesta. Las preguntas realizadas fueron las siguientes:

1. ¿Siente algún tipo de dolor en los implantes?

2. ¿Tiene algún problema cuando habla?

3. ¿Se atreve a reír con esta prótesis?

4. ¿Puede comer cómodamente?

5. ¿Está satisfecho en general con el tratamiento recibido y el resultado de éste?

6. ¿Repetiría el tratamiento?

Para las dos primeras preguntas se otorgó la siguiente puntuación: un uno para las respuestas afirmativas, un dos para las respuestas ambiguas (aquéllas en que el paciente no respondía de forma categórica con un sí o con un no, o cuando dudaba al contestar) y un tres para las respuestas negativas. Para el resto de preguntas (de la 3 a la 6) se invirtió la puntuación: un uno para las respuestas negativas, un dos igualmente para las ambiguas y un tres para las afirmativas.

Para evaluar la satisfacción de los pacientes incluidos en el estudio primero se valoraron las preguntas de forma individual mediante porcentages y posteriormente se relacionaron las diferentes variables mediante el test de c2 (grado de significación: $\mathrm{p}<0.05)$; para cuantificar la satisfacción global se sumaron los valores otorgados por los pacientes a cada pregunta y se estableció el siguiente rango:

\section{1-6: Insatisfacción \\ 7-13: Satisfacción parcial \\ 14-18: Satisfacción}

\section{RESULTADOS}

El número de pacientes edéntulos totales uni o bimaxilares intervenidos entre los años 1991 y 1998 en nuestro servicio fue de 71 , de los que 21 no pudieron ser localizados y 4 habían fallecido, por lo que el total de pacientes encuestados fue de 46 (un $64.78 \%$ del total de pacientes intervenidos).

La edad media de los pacientes era de 54.3 años (D.S. 10.0; rango 37-73 años); en los casos de edentulismo total bimaxilar la mayoría de pacientes se encontraba entre los 50 y 59 años, con una mediana de 54.5 años.

La mayoría de pacientes tratados de edentulismo total mandibular tenían entre 70 y 79 años, con una mediana de 65 años y los tratados de edentulismo total del maxilar superior entre 40 y 49 (mediana de 42.5 años) y 60 y 69 años (mediana de 60 años)(figura 1).

Del total de los pacientes encuestados 30 eran mujeres $(65.2 \%)$ y 16 eran hombres $(34.8 \%)$.

Tanto en el maxilar superior como en la mandíbula el tipo de prótesis implantosoportada colocada con mayor frecuencia fue la sobredentadura, con un $70 \%$ en el maxilar superior y un $55.3 \%$ en la mandíbula.

La mayoría de pacientes que fueron tratados de edentulismo mandibular mediante implantes, en el maxilar superior eran portadores de una prótesis completa convencional, en cambio, los pacientes que tratamos mediante implantes en el maxilar superior, en la mandíbula tenían una prótesis implantosoportada.

Haciendo referencia a los resultados de la encuesta telefónica (figura 2) nos encontramos que las tres cuartas partes de los pacientes contestaron de forma negativa a la pregunta ¿siente algún tipo de dolor en 
los implantes?. Los pacientes que contestaron afirmativamente hicieron referencia a que, de vez en cuando, notaban molestias en los implantes; otros comentaron notar "tensión" en ellos y una paciente nos comunicó que con los cambios de tiempo notaba dolor en la zona de los implantes.

La mayoría de los pacientes, un $80 \%$, no presentaban ningún problema para hablar. El resto de los pacientes se quejaron sobre todo de la dificultad de pronunciar algunos fonemas, como $\mathrm{S}$ o $\mathrm{Z}$. Un paciente comentó que al hablar se mordía y otra paciente refería tener dificultades al hablar debido a una parestesia del nervio mentoniano causada al realizarse una vestibuloplastia con injerto libre palatino en la segunda fase quirúrgica.

Prácticamente ningún paciente $(93.5 \%)$ tenía problemas para reír con su prótesis implantosoportada; solamente un paciente refirió que ésta se movía al reír.

El $82.8 \%$ de los pacientes incluidos en el estudio podían comer correctamente con su nueva rehabilitación protésica. Los pacientes que tenían algún problema a la hora de comer se referían sobre todo a la penetración de restos de comida por debajo de la prótesis, y a que ésta no era suficientemente estable.

En cuanto al grado de satisfacción general, el $65 \%$ de los pacientes estaban satisfechos con el tratamiento que se les había efectuado y con el resultado de éste.

El resto de pacientes, tanto los que no estaban satisfechos en absoluto como los que nos dieron una respuesta ambigua tenían diferentes motivos para ello, como por ejemplo que el tratamiento no cumplía con sus espectativas estéticas y/o funcionales, que no acababan de sentirse confortables con la prótesis, que la sobredentadura era demasiado sencilla, etc.

A la pregunta de si repetirían el tratamiento también el $65 \%$ de los encuestados respondió de forma afirmativa. Un $19.6 \%$ de ellos refirió que debería pensarlo mucho antes de repetirlo ya que habían tenido un postoperatorio excesivamente molesto para ellos. Los pacientes que directamente contestaron que no lo repetirían hicieron referencia a que éste era demasiado caro y largo y que comportaba demasiados sacrificios.

Si aplicamos el test de c2 podemos observar que no existen diferencias estadísticamente significativas entre el grado de satisfacción de los pacientes portadores de prótesis fija sobre implantes y de los portadores de sobredentaduras, tanto para el maxilar superior como para la mandíbula $(\mathrm{p}=0.218$ y $\mathrm{p}=0.424$, respectivamente). Tampoco existen diferencias estadístiamente significativas en la satisfacción de los pacientes si relacionamos el tipo de prótesis implantosoportada con el tipo de dentición de la arcada antagonista (tabla l).

Respecto al grado de satisfacción global de los pacientes hemos obtenido los siguientes resultados: ningún paciente presentó una puntuación inferior a 6 , sólo el $10.87 \%$ de los encuestados presentaba una puntuación entre 7 y 13 (satisfacción media) y el resto de los pacientes, el $89.13 \%$, se encontraban entre 14 y 18 , lo que según nuestro baremo de puntuación indica que sí estaban satisfechos.

\section{DISCUSIÓN}

Si para cuantificar la satisfacción utilizamos la suma de las diferentes respuestas podemos ver que el $89.13 \%$ de los pacientes edéntulos totales tratados en nuestro servicio se encontraban satisfechos; sin embargo sólo el $65 \%$ de ellos respondió de forma afirmativa a las preguntas referentes a su satisfacción y a si repetirían el tratamiento. Esto nos hace pensar en que quizá deberíamos haber incluido más preguntas en la encuesta, ya que seguramente existen otras variables que nos muestran el confort de los pacientes y que nosotros no hemos tenido en cuenta.

Probablemente una buena parte podrían haber mejorado su opinión si durante la cirugía se hubiesen sometido a algún tipo de sedación.

Si comparamos el grado de satisfacción de los pacientes en función del tipo de prótesis implantosoportada pudimos observar que no existían diferencias estadísticamente significativas entre la satisfacción de los pacientes portadores de prótesis fija y los portadores de sobredentadura, tanto en la arcada superior $(p=0.218)$ como en la inferior $(p=0.424)$.

Tampoco encontramos diferencias estadísticamente significativas al relacionar el tipo de prótesis con el tipo de dentición presente en la arcada antagonista; sin embargo, observamos que el grupo de pacientes portadores de sobredentaduras que presentó una mayor satisfacción, en un $47.6 \%$ de los casos, fue aquél en que en la arcada antagonista era portador de una prótesis completa convencional.

Los problemas que presentaron nuestros pacientes una vez terminado el tratamiento hacen referencia sobre todo a la funcionalidad de la prótesis; un $20 \%$ de los pacientes presentaban problemas para hablar y un $18 \%$ tenían dificultades para comer cómodamente. La mayoría de pacientes que no estaban satisfechos o que no repetirían el tratamiento comentaron 
que éste era largo, caro y que no cumplía con sus espectativas.

En la literatura existen diferentes trabajos (1-3,7-11) sobre la actitud de los pacientes frente al tratamiento implantológico y prostodóncico posterior. La mayoría de ellos hacen referencia a la mejoría de la calidad de vida de los pacientes que eran portadores de prótesis completas convencionales y que se habían sometido a tratamiento implantológico, o bien comparan, mediante dos grupos de estudio, a pacientes portadores de dentaduras implantosoportadas con portadores de prótesis convencionales.

En varios de estos estudios $(1,3,7,8)$ se obtuvieron resultados, en cuanto a satisfacción, superiores respecto a los nuestros; sin embargo, en nuestro caso no consideramos, en ningún momento, la prótesis completa convencional utilizada con anterioridad por parte de los pacientes, para compararla con la prótesis implantosoportada.

Wismeijer y cols. (3), en un estudio realizado en 1992, sobre la satisfacción de los pacientes portadores de sobredentaduras sobre implantes, constataron que un $95 \%$ de ellos estaban satisfechos. En este trabajo se evaluó la mejoría en la retención de la prótesis y en la autoestima de los pacientes, comparándolo cuando éstos eran portadores de prótesis convencionales.

Cibirka y cols. (7) y Grogono y cols. (8), en estudios similares, apreciaron que la satisfacción de los pacientes y su percepción subjetiva en cuanto al habla, la función, la estética y la salud bucal, con el tratamiento implantológico era superior a cuando eran portadores de una prótesis completa convencional $(p<0.0001)$. Las razones por las cuales los pacientes prefirieron el tratamiento implantológico fueron las siguientes: en primer lugar el hecho de poder mejorar su habilidad masticatoria, en segundo lugar la falta de satisfacción con las prótesis removibles previas; otra razón fue la movilidad de la prótesis convencional mandibular debida a la importante atrofia ósea (8). En el estudio realizado por Grogono y cols. (8) el $82 \%$ de los pacientes obtuvieron una mejora en su capacidad masticatoria después del tratamiento con implantes. La mayoría de los pacientes tratados con prótesis implantosoportadas refirieron notarlas como una "parte de ellos mismos" y ya no como un objeto extraño y también presentaron menos problemas en el habla. En la mitad de los pacientes también mejoró su convivencia con el sexo contrario. La mayor parte de los pacientes refirieron sentirse más seguros en su actividad social.

En el estudio efectuado por Cibirka y cols. (7) se hicieron 5 grupos de preguntas en relación al confort, la función, el habla, la estética y la imagen, compa- rándolos entre pacientes portadores de prótesis convencionales con portadores de prótesis sobre implantes. En todos los grupos de preguntas se pudo observar un aumento de las respuestas positivas a favor del tratamiento implantológico; en todos los casos el aumento oscilaba entre el 67 y el $100 \%$.

Geertman y cols. (2,9), en dos estudios en los que comparaban la satisfacción de pacientes portadores de prótesis completas y de pacientes portadores de sobredentadura, observaron en ambos casos, que los pacientes portadores de prótesis implantosoportadas estaban mayoritariamente satisfechos, pero en cambio sólo un tercio de los pacientes a los que se colocó una. prótesis convencional lo estaban. En los dos estudios, además del grado de satisfacción, también se valoró la. habilidad para masticar, comparando los dos grupos. En el primer estudio (2) se realizaron dos preguntas a cada paciente: ¿es capaz de comer un bistec? y ¿puede morder una zanahoria?. A la primera pregunta el $83 \%$ de los pacientes tratados con implantes contestaron de forma afirmativa en comparación al 32\% de los pacientes tratados con prótesis convencionales. El hecho de morder una zanahoria producía problemas al $43 \%$ de los pacientes tratados con implantes y a un $93 \%$ de los portadores de prótesis convencionales. Se realizaron dos escalas para valorar la habilidad para masticar comida blanda y dura y en ambos casos se obtuvieron resultados mejores, estadísticamente significativos $(\mathrm{p}<0.0001)$, en el grupo de pacientes tratados con una prótesis implantosoportada.

En el segundo estudio realizado por Geertman y cols. (9) también comprobaron que la eficacia masticatoria era significativamente superior en los pacientes tratados con sobredentaduras implantosoportadas que en los tratados con una prótesis completa convencional.

En los estudios en los que se incluye un grupo de pacientes que reciben tratamiento quirúrgico preprotésico $(1,10)$ la satisfacción es superior a los que reciben sólo tratamiento prostodóncico, pero nunca superior a la que presentan los pacientes sometidos a tratamiento implantológico.

Boerrigter y cols. (1) vieron, al cabo de un año del tratamiento implantológico, que el $93 \%$ de los pacientes portadores de sobredentaduras mandibulares estaban satisfechos. También el $75 \%$ de los pacientes que recibieron tratamiento quirúrgico preprotésico (vestibuloplastia y profundización del suelo de la boca) lo estaban. En cambio sólo el 38\% de los pacientes que recibieron una prótesis convencional refirieron estar satisfechos. También observaron que los dos primeros grupos presentaban una mayor habilidad masticatoria que el grupo control (prótesis convencional), grupo que en su mayoría aún presentaban problemas moderados para masticar. 
Bouma y cols. (10) en su estudio apreciaron que el grado de satisfacción y la habilidad masticatoria era superior en los grupos tratados con implantes; sin embargo, hubo una mejoría psicológica y un aumento de las relaciones sociales, al año de tratamiento, en los tres grupos.

Existen estudios que muestran resultados diferentes al nuestro. Kapur y cols. (11), en un grupo de pacientes diabéticos, no encontraron diferencias estadísticamente significativas entre la satisfacción de aquellos que eran portadores de prótesis sobre implantes y de los portadores de prótesis convencional $(72.1 \%$ y $56.9 \%$ de pacientes satisfechos respectivamente).

Los estudios publicados sobre el grado de satisfacción de los pacientes tratados con implantes $(1,3,7,8)$ obtienen resultados superiores a los nuestros. Aún así, los resultados obtenidos por Grogono y cols. (8) indican que un $89 \%$ de los pacientes repetirían el tratamiento; no obstante, sólo el $46 \%$ de ellos estaban totalmente satisfechos, siendo estos datos algo más similares a los nuestros.

\section{SUMIMARY}

The aim of this study was to evaluate the satisfaction rate of total edentolous patients treated in our service with dental implants and the placement of a posterior implant-supported prothesis (overdenture or fixed). To evaluate the satisfaction we realize a telephonic inquest with six questions: 1) Do you feel any type of pain in your implants? 2) Do you have any trouble speaking? 3) Can you laugh with your prothesis? 4) Can you eat comfortably? 5) Are you satisfied in general with the treatment recieved and with its result? 6) Could you repeat the treatment? To quantifie the satisfaction of the patients we value each answer individually and also all conjunctly. As a results, we obteined that $65 \%$ of patients were satisfied with the treatment and with its result, independently of the prothesis type they wear. Also a $65 \%$ of patients would repeat the treatment. With the $\mathrm{c} 2$ (degree of signification $\mathrm{p}<0.05$ ) we relate the different variables (type of implantosupported prothesis and type of dentition in the antagonist dental arch) with the the degree of patients' satisfaction.

\section{KEYWORDS}

Satisfaction, dental implants, overdentures and fixed prothesis.

\section{CONCLUSIONES}

1. La mayoría de pacientes sometidos a tratamiento implantológico en nuestro servicio se encuentran satisfechos.

2. No existen diferencias estadísticamente significativas entre el grado de satisfacción de los pacientes portadores de prótesis fija sobre implantes y de los pacientes portadores de sobredentaduras implantosoportadas, tanto para el maxilar superior como para la mandíbula.

3. Si relacionamos el tipo de prótesis implantosoportada con el tipo de dentición presente en la arcada antagonista tampoco hay diferencias estadísticamente significativas en la satisfacción.

4. Los factores más importantes que influyen de forma negativa en el grado de satisfacción de los pacientes son el coste económico y la duración y el esfuerzo realizado para hacer este tipo de tratamiento.

Esquema 1: Caracteristicas de un sistema de aplicación local de un antimicrobiano. 


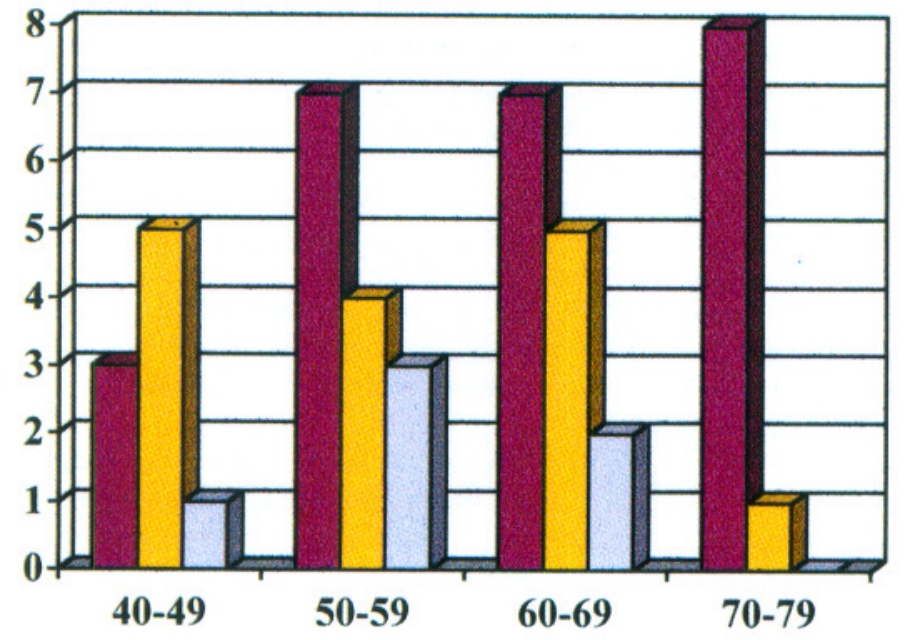

MANDÍBULA

D MAXILAR SUPERIOR

BIMAXILAR

Figura 1: Relación de la edad de los pacientes con el tipo de edentulismo tratado.

1. ¿Siente algún tipo de dolor en los implantes?

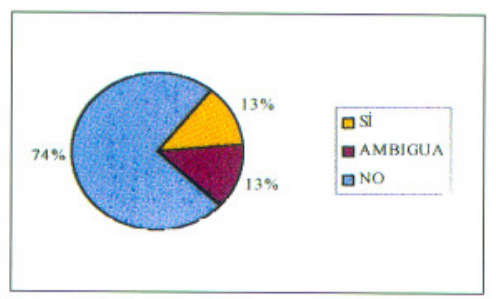

3. ¿Se atreve a reír con esta prótesis?

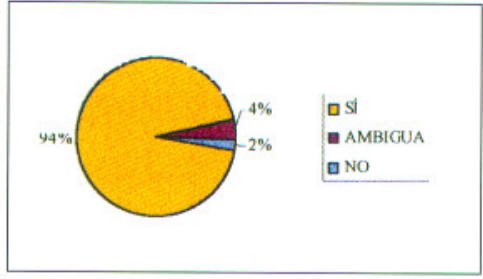

5. ¿Está satisfecho en general con el tratamiento?

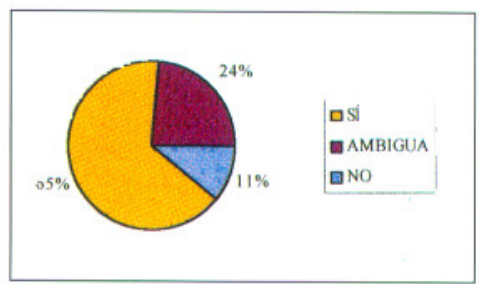

2. ¿Tiene algún problema cuando habla?

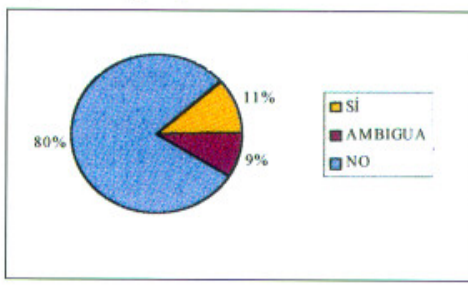

4. ¿Puede comer cómodamente?

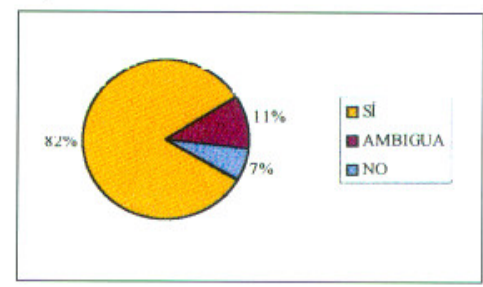

6. ¿Repetiría el tratamiento?

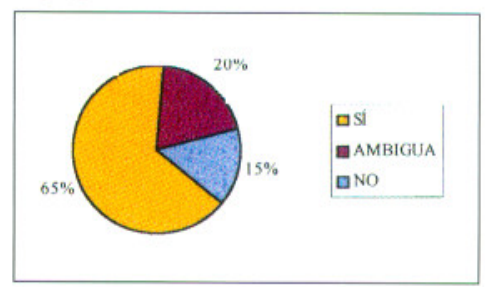




\section{BIBLIOGRAFÍA}

1. Boerrigter EM, Stegenga B, Raghoebar GM, Boering G. Patient satisfaction and chewing ability with implantretained mandibular overdentures: a comparison with new complete dentures with or without preprosthetic surgery. J Oral Maxillofac Surg 1995; 53: 1167-73.

2. Geertman ME, Boerrigter EM, van't Hof MA, van Waas MAJ, van Oort RP, Boering G, Kalk W. Two-center clinical trial of implant-retained mandibular overdentures versus complete dentures - chewing ability. Community Dent Oral Epidemiol 1996-;24: 79-84.

3. Wismeijer D, Vermeeren JIJH, van Waas MAJ. Patient satisfaction with overdentures supported by one-stage TPS implants. Int J Oral Maxillofac Implants 1992; 7: 51-5.

4. Ekfeld A, Johansson LA, Iaksson S. Implant-supported overdenture therapy: A retrospective study. Int J Prosthodont 1997; 10: 366-74.

5. De Grandmont P, Feine JS, Taché R, Boudrias P, Danohue WB, Tanguay $\mathrm{R}$, et al. Within-subject comparisons of implant-supported mandibular prothesis: psychometric evaluation. J Dent Res 1994; 73. 1096-104.

6. Feine JS, De Grandmont P, Boudrias P, Brien N, LaMarche C, Taché R, et al. Within-subject comparison of implantsupported mandibular prothesis: choice of prothesis. J Dent Res 1994; 73: 1105-11.
7. Cibirka RM, Razzoog M, Lang BR. Critical evaluation of patient responses to dental implant therapy. J Prosthet Dent 1997; 78: 574-81.

8. Grogono AL, Lancaster DM, Finger IM. Dental implants: a survey of patients' attitudes. J Prosthet Dent 1989; 62: 573-6.

9. Geertman MW, van Waas MAJ, van't Hof MA, Kalk W. Dentures satisfaction in a comparative study of implantretained mandibular overdentures: a randomized clinical trial. Int J Oral Maxillofac Implants 1996; 11: 194-200.

10. Bouma J, Boerrigter L, van Oort RP, van Sonderen E, Boering G. Psychosocial effects of implant-retained overdentures. Int J Oral Maxillofac Implants 1997; 12: 515-22.

11. Kapur KK, Garrett NR, Hameda Mo, Roumanas ED, Freymiller E, Han T, et al. A randomized clinical trial comparing the efficacy of mandibular implant-supported overdentures and conventional dentures in diabetic patients. Part I: Methodology and clinical outcomes. J Prosthet Dent 1998; 79: 555-69. 